\title{
EMBEDDED CLUSTERS IN GIANT EXTRAGALACTIC H II REGIONS: \\ II. EVOLUTIONARY POPULATION SYNTHESIS MODEL
}

\author{
Y. D. Mayya ${ }^{1}$ \\ Indian Institute of Astrophysics, Bangalore 560034 INDIA \\ Infra Red Astronomy Group, Tata Institute of Fundamental Research, Homi Bhabha Road, Colaba, Bombay 400 \\ 005 INDIA
}

\begin{abstract}
A stellar population synthesis model, suitable for comparison with Giant Extragalactic H II Regions (GEHRs), is constructed incorporating the recent developments in modelling stellar evolution by Maeder and co-workers and stellar atmospheres by Kurucz. A number of quantities suitable for comparison with broad band data of GEHRs in visible and near infrared parts of the spectrum are synthesized in addition to the hydrogen and helium ionizing photon production rates at solar metallicities, for three scenarios of star formation - (i) Instantaneous burst (IB) (ii) Continuous star formation (CSF) and (iii) Two bursts of star formation, with the older burst rich in red supergiants. For IB case, evolution of colors shows three distinct phases - an initial steady blue phase, followed by a red bump (5-15 Myr) and another steady phase with colors intermediate to the earlier two phases. CSF colors asymptotically reach peak values at $\sim 10 \mathrm{Myr}$, never reaching the reddest IB colors. Ionizing photon production rate falls off by an order of magnitude in $6 \mathrm{Myr}$ for IB, where as it almost remains constant for CSF model. Two-burst models with burst separations $\sim 10$ Myr have properties of both IB and CSF, simultaneously producing the red IB colors and high ionizing photon rate, making such regions easily distinguishable using optical observations. Flat IMFs result in bluest colors when the massive stars are on the main sequence and reddest colors during the red supergiant phase of the evolving massive stars. Errors on the computed quantities due to the statistical uncertainties inherent in the process of star formation become negligible for cluster masses in excess of $10^{5} M_{\odot}$. Our GEHR spectra in the range $200 \mathrm{~nm}$ to $3 \mu \mathrm{m}$ are found to be in good agreement with the computations of Mas-Hesse and Kunth (1991).
\end{abstract}

${ }^{1}$ Presently at the Bombay address $\quad$ E-mail: ydm@tifrvax.tifr.res.in 


\section{Introduction}

External galaxies offer excellent opportunities for the study of star formation under a variety of physical conditions. Many of the external galaxies experience a much higher level of star formation activity compared to our own Galaxy. Regions of current star formation are concentrated around the nucleus in starburst and Sérsic-Pastoriza galaxies (Balzano 1983, Sérsic \& Pastoriza 1965), while they are spread over the entire disks in blue compact dwarfs, also known as H II galaxies (Terlevich et al. 1991). Giant extragalactic $\mathrm{H}$ II regions scattered over the disks of irregular galaxies and arms of spiral galaxies are the other regions associated with intense on-going star formation activity. At the presently achievable spatial resolutions, individual stars in most of the active star-forming regions cannot be resolved or counted, owing to their greater distances. Thus, information regarding the stellar population and the star formation history in these regions has to be obtained from the integrated properties of all the embedded stars. Such a study would involve a comparison of synthesized properties of star-forming regions with observables. Most of the early efforts in this direction concentrated on synthesizing integrated colors of galaxies (Larson \& Tinsley 1978, Searle et al. 1973), which are dominated by old low mass stars (age $\sim 10^{10} \mathrm{yr}$ ). Bruzual \& Charlot (1993), Buzzoni (1989) synthesize integrated spectra of galaxies, which again sample populations averaged over lifetimes of galaxies. The effect of active star formation lasts at most for a few tens of millions of years, with significant variations over period of fractions of million year (Myr). Thus it is important to have time steps of the order of fractions of Myr to obtain a complete picture of the early evolution of an active star-forming region. Extensive grid of models covering a variety of observables in selected bands from ultraviolet (UV) to near infrared (NIR) have been synthesized by Rieke et al. (1980), Lequeux et al. (1981), Melnick et al. (1985), Olofsson (1989), Mas-Hesse \& Kunth (1991) and more recently by Leitherer \& Heckman (1995), all suitable for young star forming complexes.

In all the above models a giant star-forming complex is assumed to consist of a star cluster with an associated nebulosity around it. Ionizing photons from hot OB stars of the star cluster are the source of ionization of the nebula. Majority of the emission lines from the complex are produced in the gaseous nebula, while the UV-optical continuum has contribution from both stars and ionized gas. The dust particles embedded inside the $\mathrm{H}$ II regions absorb a fraction of ionizing photons, re-emitting them in far in- frared wavelengths. The models from different groups differ mainly in the input data, and in the slightly different synthesis techniques used. There are two main approaches adopted in the literature for synthesizing observable quantities from star-forming regions. Bruzual \& Charlot (1993) and Mas-Hesse \& Kunth (1991) among others have used observed stellar quantities as input data base, while Buzzoni (1989) and Melnick et al. (1985) have used theoretical atmospheric models of Kurucz (1979) as input. Although the former method is devoid of uncertainties in the model atmospheres, it suffers from the incompleteness in the stellar input data base, especially for the fast evolving evolutionary stages. In the present work, we follow the second method, which gives the freedom to study the active star-forming regions over a variety of physical conditions. The main aim of the present model is to synthesize the spectra and selected observable quantities in the optical and NIR regions, incorporating the latest developments in modelling stellar evolution and stellar atmospheres. Specifically the computations are done to compare the observables in the optical bands obtained through CCD imaging. The most important of these quantities is the ratio of $\mathrm{H} \beta$ to blue band luminosity. This ratio resembles the $\mathrm{H} \beta$ equivalent width, but unlike equivalent widths, it can be obtained accurately through imaging observations. It should be noted that $\mathrm{H} \beta$ equivalent widths are normally obtained through slit spectroscopy, with slits often small compared to the spatial extent of the giant star-forming complexes. This necessitates the computations of quantities suitable for imaging observations.

We are considering the phase of the starburst in which the most recent one or two bursts dominate the emission in all the bands from UV to NIR. This phase corresponds to ages of the order of a few times $10^{7} \mathrm{yrs}$. We consider three scenarios of star formation, and evolve the cluster up to $20 \mathrm{Myr}$ after the epoch of star formation. In the first scenario, all the cluster stars are assumed to have formed simultaneously (Instantaneous Burst). Stars are assumed to be forming continuously (Continuous Star Formation) in the second scenario. Third scenario we considered has a second burst occurring in the star formation complex before the death of low to intermediate mass stars from the earlier burst (multiple bursts).

In section 2, we define the model star-forming complex, and describe the methods used for synthesizing the stellar quantities. The section also includes the comparison with observed stellar quantities. The results of the computations for the three scenarios discussed above is presented in detail in section 3, while the effect of statistical uncertainties inherent in the 
process of star formation are discussed in section 4 . The computed quantities are compared with the results from earlier work in section 5. Main results are summarized in section 6 .

In paper I (Mayya 1994) of this series we reported photometric data in $B V R \mathrm{H} \alpha$ bands for around 180 $\mathrm{H}$ II complexes in nine galaxies. In the third and concluding paper in this series we will derive parameters related to star formation by comparing the observational data of Paper I with the synthesis results of this paper.

\section{Description of Synthesis Technique}

The present model assumes the star forming complex to be a star cluster surrounded by an ionized medium. Its spectrum is thus obtained by adding the spectrum of an ionized nebula to a pure cluster spectrum. This section deals with the description of the synthesis technique used in computing these two spectra.

\subsection{Definition of a Cluster}

A star cluster in the model is defined by the following set of parameters :

1. Initial Mass Function (IMF) of stars defined by

(a) Slope $(\alpha)$

(b) Upper cut-off $\left(m_{u}\right)$

(c) Lower cut-off $\left(m_{l}\right)$

2. Age $(t)$

3. Metallicity $(Z)$

4. Total Mass $\left(M_{T}\right)$

The interesting range of above parameters are, $m_{u}=30$

$120 M_{\odot}, \alpha=1.0-3.5, t=0-20 \mathrm{Myr}, Z=0.001-0.04$

$\left(Z=0.02\right.$ corresponds to solar metallicity, $\left.Z_{\odot}\right)$.

Lower cut-off mass $m_{l}$ can have any value greater than

$0.1 M_{\odot} . M_{l}$ is one of the most difficult parameters to be evaluated, because of the dominance of flux from higher mass stars in any wavelength region of observation. However, a significant fraction of the total mass of star-forming regions is locked up in low mass stars and hence the total mass as well as star formation rates are critically dependent on $m_{l}$. Based on a number of observable quantities, Rieke et al. (1980) found $m_{l}$ to be in the range $2-3 M_{\odot}$ in starburst regions. In the years that followed, there has been a number of studies favoring $m_{l}$ values in the range 1 $10 M_{\odot}$ (see Scalo 1987 for a discussion). We assume a value of $1 M_{\odot}$ for $m_{l}$ throughout the present work. Effect of changing $m_{l}$ on the computed quantities is discussed in sec. 3.1. Following formulations are used in the computations of the spectral energy distribu- tion and luminosity in any band from a star cluster.

$$
L_{\lambda}(i m f ; t ; Z)=A \int_{m_{l}}^{m_{u}} l_{\lambda}(m ; t ; Z) m^{-\alpha} d m
$$

and

$$
L_{\mathrm{B}}(i m f ; t ; Z)=A \int_{m_{l}}^{m_{u}} l_{\mathrm{B}}(m ; t ; Z) m^{-\alpha} d m
$$

with

$$
\begin{aligned}
M_{T} & =\quad A \int_{m_{l}}^{m_{u}} m^{1-\alpha} d m \\
l_{\lambda}(m ; t ; Z) & =\pi f_{\lambda}(m ; t ; Z) 4 \pi R^{2}(m ; t ; Z)
\end{aligned}
$$

where $f_{\lambda}(m ; t ; Z)$ is the intensity emitted from a star of mass $m$ at an evolutionary stage $t$ at wavelength $\lambda$. $R(m ; t ; Z)$ is the radius of the star, $A$, the normalizing constant. $Z$ is the abundance of the gas from which the stars are formed. The stellar luminosity in any given band $B$ with a normalized response curve $R_{B}(\lambda)$ is computed as,

$$
l_{B}(m ; t ; Z)=\int_{\text {band }} l_{\lambda}(m ; t ; Z) R_{B}(\lambda) d \lambda .
$$

Lyman continuum photon rate is obtained by integrating the spectrum between $22.8 \mathrm{~nm}$ and $91.2 \mathrm{~nm}$. Thus,

$$
n_{L}(m ; t ; Z)=\int_{22.8}^{91.2} l_{\lambda}(m ; t ; Z) \frac{\lambda}{\mathrm{hc}} d \lambda
$$

with h and c being Planck's constant and speed of light respectively. The above equations assume the cluster to be sufficiently massive so that masses can be considered to be continuously populated, instead of discretization into mass bins or spectral types. Under these conditions, numerical integration can be performed rather than summing over all the mass points. In sec. 4, we evaluate the minimum mass of the cluster above which numerical integration rather than summation over mass is justifiable.

\subsection{Input Stellar Data}

The computations require a library of stellar spectra for a wide range of masses at various evolutionary stages at the metallicities of interest. We used the theoretically computed stellar spectra as input dataset to our models. These spectra are synthesized by combining the stellar evolutionary models from Maeder and co-workers and stellar atmospheric models from Kurucz. We have used the evolutionary tracks from Schaller et al. (1992), which is their most recent model for $\mathrm{Z}=Z_{\odot}$. These grids differ from the earlier grids from the same group (Maeder \& Meynet 
1989, Maeder 1990) mainly in having incorporated the new radiative opacities from Rogers \& Iglesias (1992). The models assume convective core overshooting distance of 0.20 times the pressure scale height, and a metallicity dependent mass loss rate $\left({ }^{*} M \sim Z^{0.5}\right)$. For masses greater than $20 M_{\odot}$, the authors have tabulated evolutionary results for two values of the massloss rate, one being twice the other. The latter models are computed with the intention of reproducing the properties of the low luminosity Wolf-Rayet stars. As we are not aiming to count the number of WolfRayet stars as the cluster evolves, the two mass-loss schemes are expected to give identical results. We have used the set with lower mass-loss rates, which is more standard of the two. Effective temperatures for Wolf-Rayet stars in these models have been computed taking into account the extended atmospheres as described in Kudritzki et al. (1989). Massive star evolution is carried out until the end of core carbon burning in these models.

The atmospheric models of Kurucz (1979) also have been recently updated (Kurucz 1992). Apart from including many more layers than before to improve the numerical accuracy of the computations, the new models have a resolution of 1-2 nm in UV and optical regions of the spectrum. New computations are available at 1221 wavelength points from 9 $\mathrm{nm}$ to $160 \mu \mathrm{m}$. The effective temperature $\mathrm{T}_{\text {eff }}$ range of 3500-50000 has been covered for logarithm surface gravity $(\log g)$ values of 0.0 to 5.0. Kurucz grids are adequate for all stages during the evolution of stars in the mass range 1-120, except in the following situations. Effective temperature of main sequence stars more massive than $80 M_{\odot}$ (earlier than O4) exceed $50000 \mathrm{~K}$. These very hot models do not differ much from blackbody and hence we used blackbody equations when ever the temperature exceeded $50000 \mathrm{~K}$. At the low temperature end, during the fast evolving phase of red supergiants, the temperature drops below $3500 \mathrm{~K}$, for initial masses $m<20$ at ages $>10 \mathrm{Myr}$. During this phase the $\log g$ values also cross the lower limit of 0 in Kurucz grids. In these cases we have used the $3500 \mathrm{~K}$ Kurucz models with $\log g=0$. This approximation will not affect our computations for ages $<10$ Myr. Beyond this age, small effects might be seen in the NIR.

\subsection{Computational details}

Using Schaller et al. (1992) evolutionary models, we have computed $\log g$ and $T_{\text {eff }}$ at the 51 tabulated evolutionary stages for $Z=Z_{\odot}$ and $1 \leq m \leq 120$. The spectrum for a given $\log g$ and $T_{\text {eff }}$ is computed using Kurucz (1992) atmospheric models at $Z=Z_{\odot}$, interpolating the spectrum linearly in $T_{\text {eff }}$ and $\log g$ when necessary. The absolute luminosities $l_{\lambda}$ are computed from these spectra by using the mass-radius relationship as derived from the tables of Schaller et al. (see eq. 4). This results in a spectrum sampled at all the 1221 Kurucz wavelength points.

It can be seen from equations 1 and 2 that computations of cluster quantities require a knowledge of $l_{\lambda}\left(\right.$ or $l_{\mathrm{B}}$ ) at every mass point chosen for integration. Schaller et al. tabulate the evolutionary grids for 21 mass points in the mass range $0.8-120 M_{\odot}$, which is inadequate for a complete integration over the chosen mass limits. Thus it is desirable that the luminosities $l_{\lambda}$ are computed at sufficient number of mass points to represent every phase during the evolution of the cluster. This necessitated the generation of tracks for stellar masses intermediate to the tabulated masses. After considerable experimentation we found it necessary to extend the 21 mass Schaller et al. grids to 151 mass grids. Of these 102 mass points lie between $10-40 M_{\odot}$, which is the most important mass range for evolutions upto $20 \mathrm{Myr}$. Sampling interval is smoothly increased from 0.2 to $0.5 M_{\odot}$ in this mass range. For smaller number of grids the evolutionary results are found to be sensitive to the exact choice of the mass points. The interpolation scheme adopted in generating the evolutionary tracks for any intermediate mass is as follows. For a given mass, tracks for two adjacent masses from Schaller et al. are identified. A track is characterized by the mass, effective temperature $\left(T_{\text {eff }}\right)$ and bolometric luminosity $\left(l_{\text {bol }}\right)$ and is sampled at 51 evolutionary stages. New tracks for intermediate masses are generated by linear interpolations in $\log \left(T_{\text {eff }}\right)$-mass and $\log \left(l_{\text {bol }}\right)$-mass planes at all the 51 evolutionary stages. The tracks so obtained are sampled at $0.1 \mathrm{Myr}$ interval up to $20 \mathrm{Myr}$ by linear interpolations in $\log \left(T_{\text {eff }}\right)$-age and $\log \left(l_{\text {bol }}\right)$-age planes. The chosen time step is suitable enough to represent all the short-term stellar evolutionary phases. The band luminosities $l_{B}(m ; t ; Z)$ are further interpolated at mass intervals of 0.05 dex for $m<40 M_{\odot}$ and 0.2 dex for higher masses to carry out the numerical integrations. Interpolations are done linearly in $\log \left(l_{B}\right)$ - $\log (m)$ plane.

The interpolation scheme described above is adopted after visually verifying the linearity of the interpolated quantities in the plane of interpolation.

\subsection{Synthesized Quantities and Comparison with Observations}

Spectral energy distribution covering wavelengths in UV, optical and NIR regions for any given stellar mass is one of the basic quantities synthesized in our model. Luminosities in BVRIJHKLM bands 
and the number of hydrogen and helium ionizing photons are synthesized from these spectra using the eqs in sec. 2.1. The response curves needed are taken from Bessell (1990) for BVRI bands and Bessell \& Brett (1988) for JHKLM bands. Band luminosities are transformed into colors using the zeropoints derived from the Vega spectrum of Kurucz (1992), assuming Vega to have colors $0.00 \mathrm{mag}$ in all bands. Zeropoints are also derived using the observed Vega spectrum for $B V R I$ bands and model spectrum of Dreiling \& Bell (1980) for JHKLM bands. The zeropoints derived from these two methods agree within 0.01 mag. Lyman continuum photon rate is obtained by integrating model spectra shortward of $91.2 \mathrm{~nm}$ as given in Eq. 6. Similarly the helium ionizing luminosity is computed by integrating spectra shortward of $50.4 \mathrm{~nm}$. The absolute luminosity scale in the model is tied to Schaller et al. (1992) models. This approach does not require the use of the highly uncertain $T_{\text {eff- }}$ Bolometric Correction(BC) relation, to set the luminosity scale of the model. On the other hand, we derive the $\mathrm{BC}$ over the region of interest in $\mathrm{T}_{\text {eff }}$ and $\log g$ in order to compare with the observed values.

Before discussing the computations of cluster quantities, we compare the computed model colors and BCs with observed values for different spectral types and luminosity classes. The most homogenous set of stellar photometric data in BVRIJHKL bands are due to Johnson (1966). Flower (1977) has updated the $\mathrm{T}_{\mathrm{eff}}: B-V: \mathrm{BC}$ scales making use of the most recent photometry available at that time, and found Johnson (1966) scale to be in general agreement in most cases, with $B-V$ colors redder by around 0.12 mag for giants. For comparison with the model results we used $B-V$ and $\mathrm{BC}$ relations with $\mathrm{T}_{\text {eff }}$ from Flower and the rest of the photometry from Johnson. Johnson $V-R$ colors are transformed into Cousins system using the transformation equations given by Bessell (1979) (after flipping the mis-printed sign of the additive constant for the redder color range: Bessell, private communication). There is more data available in the literature for the evolving cool and hot phases of the stars (Humphreys \& McElroy 1984; Elias et al. 1985), which are in general agreement with the compilations of Johnson (1966) and Flower (1977).

Lyman continuum photon production rate per unit surface area $\left(n_{\text {lyman }}\right)$ from our computations is compared with that from Mas-Hesse \& Kunth (1991) in Fig. 1(a). Other computed quantities such as bolometric correction (BC), $B-V, V-R$ and $V-K$ colors are compared with the observed values in Fig. 1(b)(e) as a function of $T_{\text {eff }}$. In each of these figures, the observed values are denoted by filled circles for main sequence stars and inverted triangles for supergiants.
Main sequence model values are shown by the solid lines, whereas the dashed line represents the supergiant values as derived from our computations. It can be seen that on the whole, there is good agreement between our model computations and observations. Our $\mathrm{T}_{\text {eff }}: B-V$ scale for supergiants is around $0.03 \mathrm{mag}$ bluer than that of Flower (1977), with good agreement for main sequence stars. The differences are of the order of $0.1 \mathrm{mag}$ for red supergiants below 10000 $\mathrm{K}$, where the color- $\mathrm{T}_{\text {eff }}$ relation is very steep. $5-10 \%$ errors in $\mathrm{T}_{\text {eff }}$ for a given color can easily explain these differences. $V-R$ and $V-K$ show similar trends, with the model colors redder by as much as 0.2 and 0.5 mag respectively for cool red supergiants. The observed BC for main sequence and supergiants lie on either side of our computations, with the differences of the order of $0.2 \mathrm{mag}$ for $\mathrm{T}_{\text {eff }}$ greater than 10000 K. For the coolest red supergiants the model BCs are higher (less negative) by as much as $0.5 \mathrm{mag}$. As in the case of $B-V, 5-10 \%$ shift of model $\mathrm{T}_{\text {eff }}$ scale towards the cooler side for a given color can explain the observed differences in $V-R, V-K$ and BCs. Such shifts in $T_{\text {eff }}$ are not unreasonable considering the different ways in which $T_{\text {eff }}$ can be defined for extended atmospheres of stars, especially supergiants (Baschek et al. 1991). The Lyman continuum rates agree within 0.05 dex for hot main sequence stars, which mainly contribute to the total ionizing photons in a cluster. The observed differences at lower temperatures where the Lyman continuum steeply decreases with $\mathrm{T}_{\text {eff }}$ are thus not much significant.

In our model, the observational quantities are parameterized in terms of mass and hence the absolute luminosities depend on the mass-luminosity relation. As mentioned earlier, our scale is based on stellar evolutionary models of Schaller et al. (1992), which are found to be in good agreement with the values tabulated by Lang (1992) for main sequence stars. Similar comparison for supergiants could not be performed because of the uncertainty in the mass estimates for the model supergiants. However model derived $\mathrm{T}_{\text {eff- }}{ }^{-}$ luminosity relation agrees with the observed positions of red supergiants in the Hertzsprung-Russel diagram (Humphreys \& McElroy 1984). Total Lyman continuum photon production rate for high mass main sequence stars agrees within 0.1 dex with the computations of Mas-Hesse \& Kunth (1991), Leitherer (1990) and Panagia (1973). Our values begin to get systematically different (lower) at masses lower than $40 M_{\odot}$, with differences reaching a factor of 2 for $20 M_{\odot}$. The ionizing photon rate is lower by about a factor of ten for these lower masses compared to those from the hottest stars.

The basic stellar quantities and computed lumi- 
nosities for the selected stellar masses at zero age are given in Table 1(a) for solar metallicity. Table 1(b) lists the same quantities during the red and blue supergiant phases of the stars. The latter table contains quantities which are obtained by taking the weighted averages over the supergiant phases. The quantities tabulated in the two tables are:

Mass, $M_{m s}$ : Zero age main sequence (ZAMS) mass of the star

$\mathrm{M}_{\mathrm{sg}}$ : Mass of the star during its supergiant phase

$T$ : Time spent in main sequence phase (Tab. 1a) and supergiant phases (Tab. 1b)

$\mathrm{T}_{\text {eff }}, \mathrm{BC}, \mathrm{rad}, \mathrm{M}_{\text {bol }}$ : Effective temperature, Bolometric Correction, radius and bolometric magnitude of stars

$\frac{M}{L_{V}}$ : Ratio of stellar mass to the luminosity in the visual band, both expressed in solar units. $M_{m s}$ is used as mass of the supergiants rather than the highly mass-loss dependent $\mathrm{M}_{\mathrm{sg}}$.

$N_{L}$ : Number of ionizing photons emitted from the star per second

$f_{\mathrm{He}}$ : Fraction of lyman continuum photons $(\lambda \leq$ $91.2 \mathrm{~nm})$ available for the ionization of helium also $(\lambda \leq 50.4 \mathrm{~nm})$

$\frac{\phi}{L_{B}}$ : Ratio of the expected $\mathrm{H} \beta$ luminosity from the nebula surrounding the star to the blue band luminosity. See sec. 3.1 for an exact definition of this quantity. $\frac{\phi}{L_{B}}, f_{\mathrm{He}}$ and $N_{L}$ are meaningful only for very hot stars $\left(\mathrm{T}_{\text {eff }}>25000 \mathrm{~K}\right)$

colors : Optical and NIR broad band colors.

\subsection{Computations of Nebular Flux}

Ultraviolet photons from hot stars in the cluster ionize the surrounding gas leaving hydrogen, helium, oxygen, nitrogen, carbon and other astrophysically abundant elements in different ionized states. For ionization bounded nebulae, all of these ionizing photons are used up by hydrogen, and at ionization equilibrium, every ionization is followed by recombinations to one of the levels in the hydrogen atom (Osterbrock 1989). In thermal equilibrium, the energy input to the nebula in the form of kinetic energy of the electrons is lost due to collisions and radiative recombinations, leaving the nebula at a fixed temperature. This heated gas along with the ions in various excited states gives rise to a spectrum characteristic of H II regions. The basic physics governing the emission mechanism from these regions is well understood and hence its theoretical spectrum can be synthesized from only a few input parameters such as temperature, density, abundance of elements and absolute flux in one of the Balmer lines. In the next two subsections, we describe the method we have followed in synthesizing the continuum and line spectrum of the nebula.

\subsubsection{Estimation of the Gaseous Continuum}

The main emission mechanisms contributing to continuum emission from a gas are free-free, freebound, and two-photon $(2 q)$ emissions from hydrogen and helium. The net emission coefficient $\gamma_{\text {eff }}$ can be expressed as a sum of these emission coefficients, weighted by the relative abundance of the particular ionized state.

$\gamma_{\mathrm{eff}}=\gamma(\mathrm{H} \mathrm{I})+\gamma(2 \mathrm{q})+\frac{n\left(\mathrm{He}^{+}\right)}{n(\mathrm{H})} \gamma(\mathrm{HeI})+\frac{n\left(\mathrm{He}^{++}\right)}{n(\mathrm{H})} \gamma(\mathrm{HeII})$,

where $n(\mathrm{H})$ is the total number density of hydrogen atoms in both ionized and neutral states. In our computations we assume a helium abundance of $10 \%$ by number all of which is in the singly ionized state. Individual $\gamma$ 's as computed and tabulated by Brown \& Mathews (1970) are used here for $\lambda<1 \mu \mathrm{m}$. For longer wavelengths computation of Ferland (1980) have been used. The continuum of the gas spectrum in erg s $\mathrm{s}^{-1} \AA^{-1}$ is related to $\gamma_{\mathrm{eff}}$ by,

$$
\mathrm{L}_{\lambda}^{\text {neb }}=\gamma_{\text {eff }}\left(\frac{c}{\lambda^{2}}\right)\left(\frac{1}{\mathrm{E}_{\mathrm{H} \beta} \alpha_{\mathrm{H} \beta}^{\mathrm{eff}}\left(\mathrm{H}^{0}, T_{e}\right)}\right) \mathrm{L}_{\mathrm{H} \beta}
$$

$\mathrm{E}_{\mathrm{H} \beta}$ is the energy of the $\mathrm{H} \beta$ photon, $\alpha_{\mathrm{H} \beta}^{\mathrm{eff}}\left(\mathrm{H}^{0}, T_{e}\right)$ is the $\mathrm{H} \beta$ recombination coefficient and $\mathrm{L}_{\mathrm{H} \beta}$ is the $\mathrm{H} \beta$ line luminosity in erg $\mathrm{s}^{-1} . T_{e}$ is the temperature of the nebula at thermal equilibrium.

\subsubsection{Estimation of the Gaseous Emission line Strengths}

Osterbrock $(1974,1989)$ has compiled the emission line strengths of various transitions of hydrogen and helium relative to $\mathrm{H} \beta$ line strength for typical densities and temperatures of $\mathrm{H}$ II regions. The strength of emission lines from other elements however are dependent on the relative abundance of the elements, the state of ionization, the temperature structure of the nebula etc. and hence their computations require detailed modelling. Such models have been constructed by Dopita \& Evans (1986), Rubin (1985), McCall et al. (1985) among others. From observations of a large sample of extragalactic $\mathrm{H}$ II regions McCall et al. have shown that the majority of the emission line ratios can be parameterized by a single parameter, namely $\frac{\mathrm{I}[\mathrm{O} \mathrm{II}]+\mathrm{I}[\mathrm{O} \mathrm{III}]}{\mathrm{I}(\mathrm{H} \beta)}$. The basic physics behind this can be summarized as follows. The cooling of the nebula is mainly controlled by the radiative losses in oxygen ions, which constitute more than $45 \%$ (Lequeux et al. 1979) of metallic mass. This gives rise to an 
anti-correlation between oxygen abundance and nebular temperature. Most of the oxygen in GEHRs is in singly ([O II $]$ ) and doubly ([O III $]$ ) ionized states, both of which have bright emission lines in the optical region ([O II $] \lambda 3726$ and [O III $] \lambda 5007)$. Hence, oxygen abundance can be quite accurately estimated using one or both of these lines. The observed correlations of line ratios of other elements with oxygen abundance suggests that the relative abundance of other elements such as C, N and S do not vary much from region to region. Photoionization models are successful in reproducing the observed correlation between line ratios and oxygen abundance, enabling the prediction of line ratios from a knowledge of oxygen abundance alone.

Our interest here is to estimate the contribution of emission line fluxes to $B V R$ band fluxes. We considered all the bright lines of $\mathrm{N}, \mathrm{O}$ and $\mathrm{S}$ apart from hydrogen lines as computed by McCall et al. (1985). Their tables 15 and 12 help us to estimate the oxygen abundance from the strengths of [O II $] \lambda 3726$ and [O III] $\lambda 5007$ lines, which in turn are used to estimate the strength of various lines from other elements.

The nebular spectrum is then obtained by adding selected emission lines to the continuum spectrum. Nebular spectrum when added to the cluster spectrum results in a spectrum expected from a starforming complex.

\section{Star Formation Scenarios}

We consider three possible scenarios of star formation which might be at work in GEHRs. In the simplest case referred as Instantaneous Burst (IB), all the stars are assumed to have formed over a short time scale (coeval) compared to the lifetime of even the most massive stars. The other extreme case is to assume the star formation to be continuous over a period of time with a star formation rate (SFR), which is either a constant (CSFR) or exponentially decreasing (ESFR) function of time. Thirdly we consider an intermediate scenario between the two mentioned. We model the effect of two instantaneous bursts separated over time scales comparable to the lifetime of the stars produced in the burst. Thus at any given time, there will be stars from two generations. We refer to this model as Multiple Bursts (MB). The results from these three models are described separately in the following sections.

\subsection{Instantaneous Burst of Star Formation}

The IB of star formation is fully described by the equations in sec. 2.1. All the quantities synthesized for individual stars are also synthesized for clusters.
The dependence of the computed quantities on the IMF parameters for a zero-age cluster is presented in Tab. 2(a). Model numbers are given in the first column. The IMF parameters, namely the upper cut-off mass and slope are given in columns 2 and 3. Ratios of the total mass to bolometric $\left(\frac{M}{L}\right)$ and visual $\left(\frac{M}{L_{v}}\right)$ luminosities are given in columns 4 and 5 in solar units. Lyman continuum photon production rate $\left(N_{L}\right)$ per unit mass of the cluster is given in column 6 . Fraction of the hydrogen ionizing photons which are also capable of ionizing helium are given in column 7 . Lyman continuum photon rate $N_{L}$ is also expressed in units of expected $\mathrm{H} \beta$ luminosity $\phi$ from a nebula at $10000 \mathrm{~K}$ and defined as,

$$
\phi=4.78 \times 10^{-13} N_{L} .
$$

$\phi$ in the above equation has dimensions of $\operatorname{erg~s}^{-1} M_{\odot}{ }^{-1}$, and $N_{L}$ in $\mathrm{phs}^{-1} M_{\odot}{ }^{-1}$. Column 8 contains $\frac{\phi}{L_{B}}$, which is the ratio of the expected $\mathrm{H} \beta$ to blue band luminosity. $\mathrm{H} \beta$ equivalent width is directly related to $\frac{\phi}{L_{B}}$ as can be seen from Fig. 2. The relationship is derived by evolving a cluster up to $20 \mathrm{Myr}$, with the dots on the curves separated by $0.5 \mathrm{Myr}$ interval. Column 8 contains this ratio. Columns 9-14 contain optical and NIR colors of the cluster (nebular contribution not taken into account). $\mathrm{H} \beta$ and $\mathrm{H} \alpha$ equivalent widths in angstrom are given in column 15 and 16 respectively, assuming a gas temperature of 10000 $\mathrm{K}$.

The effect of changing lower cut-off masses on the computed quantities is illustrated in Tab. 2(b), expressed as differences from an IMF with $m_{l}=1 M_{\odot}$ in logarithm or magnitude units. The tabulated values correspond to an upper cut-off mass of $60 M_{\odot}$, but have identical values for $30<m_{u}<120 M_{\odot}$. Tabulated quantities in columns $4-16$ are identical to those in Tab. 1, except in column 7 , which represents the factor by which the mass of the cluster decreases due to the change in $m_{l}$ from a value of $1 M_{\odot}$. It can be seen that the quantities which are most affected by changing the lower cut-off mass are $\log \left(\frac{M}{L}\right), \log \left(\frac{M}{L_{v}}\right)$ and $N_{L}$. Changing the lower cut-off has only the effect of changing the total mass of the cluster with negligible change in the luminosities, as can be inferred by comparing columns $4-6$ with column 7 . An IMF with a lower cut-off of $1 M_{\odot}$ contains $4-6$ times more mass compared to an IMF with $m_{l}=10 M_{\odot}$ for IMF slope $=2.5$ and $32-38$ times more for slope $=3.5$ for $m_{u}$ in the range 120 to $30 M_{\odot}$. Among colors $V-K$ is most sensitive to changes in $m_{l}$ due to nonnegligible contribution to $K$ band luminosity from low mass stars. However contribution from stars less massive than $1 M_{\odot}$ is negligible to any band luminosity and hence colors will remain the same. Quantities in 
columns $4-6$ can be reproduced for a different $m_{l}$ by adding the entries in Tab. 2(a) to the logarithm of the quantity,

$$
f_{M}=\frac{1-m_{u}^{2-\alpha}}{m_{l}^{2-\alpha}-m_{u}^{2-\alpha}} .
$$

A real giant star-forming complex differs from its definition in the model in the following ways.

1. Dust can be present inside star-forming complexes, which absorb and hence reduce the total number of photons available for ionization. Throughout their computations Mas-Hesse \& Kunth (1991) have assumed that $30 \%$ of the ionizing photons are absorbed by dust, based on the work of Belfort et al. (1987). However Mezger (1978) has shown this fraction to be highly uncertain having values anywhere between 0.2 to 0.7 depending on the local conditions. Because of the large uncertainty in the value, we prefer to present all the model values without correcting for dust absorption. We leave it as a free parameter to be assumed or determined while comparing with observations.

2. Reddening due to foreground interstellar dust and nebular continuum emission in different bands make the observed colors different from the values computed for a pure cluster. Both these effects can be handled in a better way using available information on the physical conditions of the individual regions while comparing with observations. Thus we tabulate the pure cluster values instead of taking into the effects of interstellar extinction and nebular emission in an average way.

3. Coevally formed stars take different amounts of time to reach the ZAMS. For example a star of $100 M_{\odot}$ takes only $1.9 \times 10^{4} \mathrm{yr}$, where as a star of $1 M_{\odot}$ takes 75 Myr (Ezer \& Cameron 1967, Beech \& Mitalas 1994). Schaller et al. (1992) models we have used considers the stellar evolution from ZAMS stage onwards, thus our early stage cluster evolution might be affected. Using the pre-main sequence (PMS) evolutionary tracks of Ezer \& Cameron (1967), we estimated the PMS contribution relative to the ZAMS luminosities in various bands. PMS luminosity of even the low mass stars remains within a factor of $2-3$ of the ZAMS value for more than $80 \%$ of the PMS evolutionary stage. On the other hand, in the earlier stages, cluster luminosity is dominated by the luminous massive main-sequence stars, whose bolometric luminosity is $10^{5}--10^{6}$ times that of $1 M_{\odot}$ star, rendering the inclusion of PMS stages unnecessary. Thus computations of cluster quantities based on Schaller et al. models are valid even at stages where low mass stars are yet to reach the ZAMS.

More massive a star is, earlier it exhausts the nuclear fuel and hence the evolution of the IB model of
$\mathrm{SF}$ is characterized by the selective removal of massive luminous stars from the cluster. As the massive stars wander around in the H-R diagram during their post main-sequence evolution, they affect the integrated properties of the cluster, even when their number is small. We have evolved the cluster at steps of $0.1 \mathrm{Myr}$ interval upto $20 \mathrm{Myr}$, when the highest surviving mass is $\sim 11 M_{\odot}$. A detailed discussion of the these results will be presented in sec. 3.4 along with the other two scenarios of star formation considered.

\subsection{Continuous Star Formation}

In the previous section we considered the evolution of a cluster containing coeval stars formed in an instantaneous burst. Now consider the case where stars are formed continuously resulting in stars of different ages in the same cluster. This scenario, known as continuous star formation (CSF), is characterized by a star formation rate (SFR), the rate at which new stars are added to the cluster. We have considered two kinds of SFRs, a constant rate of star formation (CSFR) and an exponentially decreasing star formation rate (ESFR).

Computationally CSF scenario is realized by summing luminosities over all surviving masses, which is mathematically represented as,

$L_{\mathrm{B}}^{\operatorname{csf}}(i m f ; t ; Z)=\frac{A \int_{0}^{t} \int_{m_{l}}^{m_{u}} l_{\mathrm{B}}\left(m ; t^{\prime} ; Z\right) m^{-\alpha} R\left(t-t^{\prime}\right) d m d t^{\prime}}{\int_{0}^{t} R\left(t^{\prime}\right) d t^{\prime}}$

with

$$
R\left(t^{\prime}\right)=\quad R_{0} \quad \text { for CSFR }
$$

and

$$
R\left(t^{\prime}\right)=\quad R_{0} \exp \left(-\frac{t^{\prime}}{t_{0}}\right) \quad \text { for ESFR }(13
$$

$R\left(t^{\prime}\right)$ represents the rate of star formation at an epoch $t^{\prime}$ with $R_{0}$ and $t_{0}$, the SFR at $t=0$ and the e-folding time of SFR respectively. The denominator in eq. 11 is a measure of total mass in all the stars and $A$ is the normalization constant defined in eq. 3 .

\subsection{Multiple Bursts of Star Formation}

We now consider the third scenario of star formation, in which a second burst of star formation takes place before the death of intermediate-low mass stars from the earlier burst. In several galactic OB associations, neighbouring regions of star formation are found to differ in age by $\sim 10 \mathrm{Myr}$, which is thought to be evidence for sequential star formation (Elmegreen 1992). The energetic events such as stellar winds, supernova activity, typical of massive stars, might be responsible for the sequential star formation 
(Elmegreen \& Lada 1977). In distant star forming regions, the resolution may not be good enough to resolve the populations spatially, and hence one has to infer the presence of more than one burst based on the integrated light. In this study we aim at providing observational plots where regions with two bursts of star formation can be identified.

Computations are done by adding the fluxes from two populations of equal strength, one younger than $6 \mathrm{Myr}$ and the other older than $6 \mathrm{Myr}$. Each populations is assumed to be independently evolving like an IB with identical IMFs.

\subsection{Discussion of Evolutionary Results}

As discussed in section 2.1, an IB cluster is defined by its age, metallicity and total mass apart from the three IMF parameters. CSF and MB scenarios have additional parameters defining them. Thus the total parameter space involved is large for full presentation of the results. It should also be noted that Mas-Hesse \& Kunth (1991) and more recently Leitherer \& Heckman (1995) have presented results for considerable section of the parameter space. In this study, we concentrate on comparison of the three scenarios of star formation for typical values of the parameters, instead of presenting results over the entire parameter space. We discuss the IB scenario in greater detail for two reasons - (i) The evolutionary trends in this case can be directly associated with the evolution of stars and (ii) The results of IB scenario can be used to explain the composite models such as CSF and MB.

As has been pointed out earlier, we have fixed the metallicities at $Z=Z_{\odot}$ and normalized the quantities to cluster mass $M_{T}=1 M_{\odot}$. An IMF with $m_{l}=1, m_{u}=60$, and slope $=2.5$, which has slope close to Salpeter's value of 2.35 for the solar neighbourhood, is chosen as a representative IMF in our computations. We refer this IMF as "standard" in rest of the article. The effects of different IMF parameters are inferred by computing quantities for two extreme IMFs - (i) $m_{l}=1, m_{u}=30$, slope $=3.5$, and (ii) $m_{l}=1, m_{u}=120$, slope $=1.0$. The first one is heavily biased against high mass stars while the second one is rich in massive stars. Comparison of the results for the three IMFs is done in sec. 3.6.

We depict the contribution from different masses to the cluster spectrum as a function of age of the IB in Figs 3(a)-(f). The mass range $1-60 M_{\odot}$, for the standard IMF is divided into four sub-intervals, namely (i) low mass stars $(1<m<5)$, (ii) intermediate mass stars $(5<m<15)$, (iii) high mass stars $(15<m<40)$ and (iv) very high mass stars $(40<m<60)$. The quantities chosen are (a) Bolo- metric luminosity, (b) Lyman continuum photon rate, (c)-(f) $B, V, R$ and $K$ band luminosities respectively. The ordinates are expressed in units of $\operatorname{erg~\mathrm {s}^{-1}} M_{\odot}{ }^{-1}$ in all figures except (b), where it is in $\mathrm{ph} \mathrm{s}^{-1} M_{\odot}{ }^{-1}$. The dotted, dashed and dash-dotted lines represent the contribution from stars in $40-60 M_{\odot}, 15-40 M_{\odot}$ and $5-15 M_{\odot}$ range respectively. The contribution from the $1-5 M_{\odot}$ bin always lies below the plotted range. The solid line in the plots is the sum of contribution from all the stars, which is plotted separately for four of the optical and NIR bands in Fig. 3(g). The bands are marked on the curves. Also marked in Fig. 3(g) are the beginning and end of the supergiant phases for the selected masses. The following conclusions can be drawn from these figures.

1. Early cluster evolution is dominated by the evolution of stars more massive than $15 M_{\odot}$, with lower mass stars beginning to contribute substantially at around 10 Myr.

2. All quantities except $N_{L}$ show an initial increase in luminosity peaking at an age between 5-8 Myr before the decline starts. The rate of increase is mainly controlled by the evolving massive stars $\left(>40 M_{\odot}\right)$. Peak luminosities are reached earlier at shorter wavelengths compared to that at longer wavelengths, as the IB cluster evolves. Lyman continuum photon rate remains steady for the first $3 \mathrm{Myr}$, decreasing monotonically then onwards.

3. The initial increase in luminosity in optical and NIR bands is due to the combined effects of the increase in bolometric luminosity and the decrease in the effective temperature of massive stars as they evolve off the main-sequence. The increase in luminosity is larger at longer wavelengths (e.g. $K$ band). 4. Bolometric luminosity and ionizing photon rates are heavily dependent on the massive luminous stars and hence decrease rapidly once these stars die at around 5 Myr. On the other hand the luminosities in optical and NIR bands decrease only marginally after the death of massive stars.

5. The peak luminosities in optical and NIR bands during the evolution of the cluster are due to the red supergiant phase of the $15-40 M_{\odot}$ stars. The red supergiant contribution to total luminosity as compared to that of the low mass main sequence stars is increasingly significant at longer wavelengths. For example $99 \%$ of the $K$ band luminosity is contributed by the red supergiants at around $10 \mathrm{Myr}$.

6 . The only regime where masses less than $5 M_{\odot}$ show their presence is in the evolution of optical luminosities beyond $12 \mathrm{Myr}$. Even here their contribution is less than $20 \%$.

7. Small wiggles in the diagram especially beyond $15 \mathrm{Myr}$ are not genuine, but are due to the limita- 
tions of the computations.

The evolutionary results for the three scenarios of star formation are presented in Tabs 3, 4 and 5. Refer Tab. 2(a) for the explanation of the column headers in these tables. Tabulated quantities are smoothed over a period of 0.5 Myr to suppress the short term fluctuations on the computed quantities. For continuous star formation model, constant SFR is assumed in the computations of the tabulated quantities. Columns 1 and 2 of Tab. 5 give the ages of the young and old bursts for the two-burst models. The data are presented for the younger bursts at ages 0, 3.5, 5.0 and 6.0 Myr and older bursts of equal strength but evolved to ages between 6 and 14 Myr. Computational results for only the standard IMF are presented in the printed form. These Tabs $3-5$, containing the results for the three IMFs discussed above are presented in AAS CD-ROM series.

The Evolution of the most important observable quantities is shown graphically in Figs 4(a)-(d) for the standard IMF. The solid lines depict the quantities smoothed over 0.5 Myr with the dotted lines representing the un-smoothed quantities at intervals of 0.1 Myr for IB scenario. Results for CSF scenario are plotted as dashed and dot-dash lines corresponding to exponentially decreasing SFR with $t_{0}=10 \mathrm{Myr}$ and constant SFR respectively. Quantities plotted are $\log \left(\frac{\phi}{L_{B}}\right), B-V, V-R$ and $V-K$ in Figs 4(a), (b), (c) and (d) respectively. $\left(\frac{\phi}{L_{B}}\right)$ drops by two orders of magnitude in $10 \mathrm{Myr}$ in the case of IB scenario. In contrast this quantity falls by only a factor two during the same time period for CSFR and by a factor of e for ESFR. For IB scenario, $\frac{\phi}{L_{B}}$ follows the evolution of $N_{L}$, which is controlled by the massive stars. The continuous creation of massive stars in CSF models keeps the $\frac{\phi}{L_{B}}$ almost steady.

Three distinct phases can be identified in the evolution of optical and NIR colors for IB. Colors remain constant at their zero age values for the first $3 \mathrm{Myr}$, during which period main sequence stars dominate the cluster evolution. The second phase is characterized by a red bump during 5-15 Myr. This phase corresponds to the appearance of red supergiants in the cluster. Colors again become steady at around $16 \mathrm{Myr}$, at a value mid-way between the blue colors of main sequence phase and the average colors of red supergiant phase. During this phase surviving stars have masses $<12 M_{\odot}$ and hence the cluster lacks luminous red supergiants. The color evolution is determined by the slowly evolving intermediate mass stars and hence colors are steadier. Larger amplitude of the dotted lines during this phase represents the limi- tations of the computations. Increasing the thickness of the mass grid for stars less massive than $12 M_{\odot}$ is required to reduce these fluctuations.

Evolution of the colors in the red supergiant phase requires a detailed discussion. $B-V$ attains the reddest value of 0.38 at $7 \mathrm{Myr}$, drops down to a value of 0.12 at $9 \mathrm{Myr}$, shows two small peaks there after before entering the third phase. $V-R$ shows the first peak at $8 \mathrm{Myr}$ reaching peak color of 0.4 , which drops down to 0.29 at 9 Myr. Notice that the drop is stronger for $B-V$ compared to $V-R$ and $V-K$. Infact $V-K$ color becomes reddest at $11.5 \mathrm{Myr}$. The position of the color peaks depends on the detailed evolution of massive red supergiants, which, when present dominate the luminosity in optical and NIR bands. Fig. 3(g) is useful in understanding the color behaviour.

Colors for CSF remain steady at zero-age values upto 3-4 Myr, increasing to values of $0.1,0.2$ and 2.0 for $B-V, V-R$ and $V-K$ colors respectively. Colors remain steady at these values after about $10 \mathrm{Myr}$. CSF colors remain bluer than the IB colors during the luminous red supergiant phase of cluster evolution. Difference between CSFR and ESFR models is very small compared to their differences from IB models.

\subsection{Cluster Evolution in Observational Plane}

So far we studied the behaviour of the observable quantities as a function of age of the cluster. Now we discuss the cluster evolution in $\log \left(\frac{\phi}{L_{B}}\right)$ vs color $(B-V, V-R$ and $V-K)$ plane. These are shown in Figs 5(a)-(c) for the standard IMF. Observed quantities can be directly plotted on these diagrams, and hence the plots are very useful for diagnosis of star formation. Dotted line denotes the locus of IB model evolved upto $13 \mathrm{Myr}$, with the thicker dots placed every 0.5 Myr interval. CSF model with a constant SFR is shown by the dashed line, covering $20 \mathrm{Myr}$ of evolution. The solid lines correspond to models with two bursts of star formation. Four sets of models are plotted. Age of the younger population is fixed for each set at $0,3.5,5$ and 6 Myr from top to bottom respectively. These ages are chosen to represent most prominent evolutionary phases of the cluster and the corresponding positions are marked by asterix on the IB model. The age of the older population varies from 6 to 14 Myr along a given set, with thick dots denoting 0.5 Myr intervals.

CSF models occupy only a narrow strip in the observational plane even after evolution upto $20 \mathrm{Myr}$. On the other hand 2-burst models cover a much larger range in $\log \left(\frac{\phi}{L_{B}}\right)$ and colors. It is interesting to note that these composite models simultaneously produce 
high $\frac{\phi}{L_{B}} \quad$ values and red colors, when the age of the younger population is $<3 \mathrm{Myr}$. Younger population controls the ordinate, while the colors are controlled by the red supergiants of the older population. This phase of cluster evolution can be easily distinguished from IB and CSF models.

\subsection{Effect of IMF on cluster evolution}

All the discussions in the previous sections were centered on the intermediate IMF. In this section we compare those results with two extreme IMFs in order to judge the effect of IMF differences on observational quantities. The high mass deficient IMF has parameters $m_{l}=1, m_{u}=30$ and $\alpha=3.5$, where as the high mass rich IMF has parameters $m_{l}=1, m_{u}=120$ and $\alpha=1$.

Evolution of $\log \left(\frac{\phi}{L_{B}}\right), B-V, V-R$ and $V-K$ for IB with the three IMFs discussed above are shown in Figs 6(a)-(d). Solid line uses the standard IMF, where as the dotted and dashed lines correspond to high mass enriched and high mass deficient IMFs respectively. The initial steady phase in $\frac{\phi}{L_{B}}$ and colors prolongs up to $5.5 \mathrm{Myr}$ for the high mass deficient IMF. $\frac{\phi}{L_{B}}$ is lower by an order of magnitude during this steady phase compared to the standard IMF due to the reduction in the number of stars contributing to ionizing photons. The red bump in the color evolution is less pronounced for this high mass truncated IMF. Note that the colors are redder during the steady phase and bluer in the red supergiant phase as compared to the standard IMF values.

Evolution with high mass enriched IMF is interesting in several respects. An examination of Fig. 3, which is for the standard IMF, is useful in understanding the details of the evolution. The initial steady phase in $\frac{\phi}{L_{B}}$ is almost absent where as it is shortened for colors. This is due to the domination of optical and NIR luminosities by stars more massive than $40 M_{\odot}$. The extent of domination is more for the high mass enriched IMF compared to standard IMF. During the early evolution optical and NIR luminosities increase rapidly where as $N_{L}$ remains steady resulting in the decrease of $\frac{\phi}{L_{B}}$. Due to the shorter lifetime of high mass stars, the cluster gets blue and red supergiants earlier on during its evolution, leading to shortening of the initial steady phase in colors. All the stars more massive than $60 M_{\odot}$ cease to exist after 3.5 Myr and hence beyond this age, the high mass enriched IMF differs from standard IMF only in having a flatter slope. Evolution of $\frac{\phi}{L_{B}}$ is almost independent of IMFs after $5 \mathrm{Myr}$. The shape of color evolution during the red supergiant phase (5-15 Myr) is only weakly dependent on IMF parameters, but the abso- lute value of colors during this phase is strongly IMF dependent. $B-V, V-R$ and $V-K$ colors are redder by $0.25,0.15$ and $0.4 \mathrm{mag}$ respectively in comparison to the standard IMF. Redder colors are due to an increase of high luminosity red supergiants with respect to low luminosity red supergiants and main sequence stars, as a result of the flatter slope. In summary, increasing the fraction of high mass stars makes the cluster colors bluer in the early phase $(<3 \mathrm{Myr})$ and redder during the red supergiant phase (5-15 Myr).

Effect of IMFs for CSF models are shown in Figs 7(a)(d). Line types and notations are as given for Fig. 6 . $\left(\frac{\phi}{L_{B}}\right)$ has a smooth evolution with high mass enriched IMF always having higher value of $\frac{\phi}{L_{B}}$. Massive star enriched IMFs have bluer colors during the first 5 Myr and redder colors beyond that compared to other IMFs. Reddest colors reached by the CSF models are not as red as that of IB models ( 0.1 vs $0.65,0.2$ vs 0.55 and 2.0 vs 3.6 for $B-V, V-R$ and $V-K$ respectively).

Two-burst model results for the three IMFs are plotted in the observable planes in Figs 8(a)-(c). The line types used to distinguish IMFs are as for the previous figures. There are four sets of models for each IMF. Burst parameters are similar to that for Fig. 5. Age of the younger population is marked on each track. The high mass enriched IMF spans a considerable range in the observable plane. The three IMFs are clearly distinguishable for younger burst ages less than 3.5 Myr. Beyond this age, they are separated only in the optical colors.

\section{Statistical Effects on the Derived Quanti- ties}

Following the normal approach, we have assumed the stellar masses to be continuous and have numerically integrated the Eqs 1 and 2 over mass. Two clusters with identical IMFs can have different distribution of the stellar masses, leading to different band luminosities, summed over all the stars. The mean of the luminosities over several clusters with identical IMFs, is however expected to be the same as that obtained using the integration approach. The departure from the mean value is dependent on the cluster mass $M_{T}$, approaching zero for massive clusters. We investigate here the minimum mass required for an assumed IMF, such that statistical fluctuations in star formation does not affect the observable quantities.

We aim to estimate the errors on the computed quantities due to the statistical selection of stellar masses in a real star forming complex, as a function of the total mass of the star cluster. This requires a knowledge of the masses of all the stars in the cluster. 
Monte Carlo method is used for simulating real star clusters satisfying the IMF with $m_{l}=1, m_{u}=120$ and $\alpha=2.5$. The IMF is normalized such that at least one star earlier than O4 $(90<m<120)$ exists in the cluster. The mass of the cluster containing only one such star is the minimum mass of the cluster for the assumed IMF and hence is referred as $M_{\min }$ - The corresponding number of stars in the cluster is $N_{\min }$. For the chosen IMF, $M_{\min }=6641 M_{\odot}$ and $N_{\min }=2434$. Cluster masses $\left(M_{T}\right)$ and total number of stars $\left(N_{T}\right)$ are defined in integer units of $M_{\min }$ and $N_{\min }$ respectively. Thus $M_{T}=n \times M_{\min }$ and $N_{T}=n \times N_{\min }$. Physically $n$ denotes the number of stars earlier than $\mathrm{O} 4$.

For a given mass $M_{T}, N_{T}$ stars with masses between $1-120 M_{\odot}$ are generated weighted by the IMF slope. The slope of the IMF for the generated mass string is re-determined and confirmed to be within 0.01 of the input value of 2.5. Luminosity in a given band $B$ from such a mass string is computed by adding the band luminosities from all the stars.

$$
L_{B}\left(i m f ; t ; Z ; M_{T}\right)=\left(\frac{1}{M_{T}}\right) \sum_{i=1}^{N_{T}} l_{B}\left(m_{i} ; t ; Z\right)
$$

where $m_{i}$ is the mass of the $i^{t h}$ star generated during the simulation. $L_{B}$ is computed at $Z=Z_{\odot}$ for $t=0-20$ Myr at intervals of $0.1 \mathrm{Myr}$. The process of generating $N_{T}$ stars and computing $L_{B}$ s are repeated for 100 trials, thus simulating 100 clusters with the same IMF and total cluster mass. The error due to the statistical selection of stars is estimated as rms noise of the 100 trials.

The above exercise is carried out for $n=3,10$ and 50. The mean values of the computed quantities over 100 trials agree well with the values obtained from the integration method. Rms errors on the representative luminosities and colors are plotted in Figs. 9 and 10 for $n=3,10$ and 50. Errors on the bolometric luminosity, Lyman continuum photon production rate, expressed in terms of $\mathrm{H} \beta$ flux, $B$ and $K$ band luminosities are plotted in Fig. 9(a)-(d) respectively. Errors on $\frac{\phi}{L_{B}}, B-V, V-R$ and $V-K$ colors are plotted in Fig. 10(a)-(d). Decrease of rms errors with increasing cluster mass (or increasing $n$ ) can be easily inferred from these plots. The errors on colors are larger during the red supergiant phase of the cluster, as compared to other phases. For $n=50$ $\left(M_{T}=3.3 \times 10^{5} M_{\odot}\right)$ the errors on $\log \left(\frac{\phi}{L_{B}}\right)$ are less than 0.03 dex throughout the 20 Myr evolution. Errors remain below $0.03 \mathrm{mag}$ for $B-V$ and $V-R$ colors during the same period. $V-K$ errors marginally exceed $0.1 \mathrm{mag}$ for ages greater than $16 \mathrm{Myr}$. The errors on $\log \left(\frac{\phi}{L_{B}}\right)$ and colors are larger by a fac- tor of $2-3$ for clusters 5 times less massive $(n=10)$. However these errors are within the various photometric error sources involved during the photometry of GEHRs (Mayya 1994). Hence for embedded clusters more massive than $\sim 10^{5} M_{\odot}$ or more luminous than $10^{39} \mathrm{erg} \mathrm{s}^{-1}$ in $\mathrm{H} \alpha$, the errors due the statistical selection of masses are negligible. For comparison, 30 Doradus nebula in the Large Magellanic Cloud has $\mathrm{H} \alpha$ luminosity of $1.5 \times 10^{40} \mathrm{erg} \mathrm{s}^{-1}$ (Mathis et al. 1985).

\section{Comparison with Other Models}

Currently a number of models exist, which synthesize the cluster parameters at various wavelength bands. Among these, the most suitable model for comparison with our results is from Mas-Hesse \& Kunth (1991). We compare our monochromatic spectrum of a cluster+nebula with their table 6(a). Comparisons are made at all the tabulated ages $(2,5,7.5$, 10 and $20 \mathrm{Myr}$ ) for IMFs with $m_{l}=2, m_{u}=120$ and $\alpha=2.0$. In their model, Mas-Hesse \& Kunth (1991) have assumed that only $70 \%$ of the available Lyman continuum photons are used up for ionization. We have taken this into account in our comparison. The results of the comparisons are given in Figs 11(a)-(e). Spectra integrated over the entire star forming complex are shown in these Figs, between $70 \mathrm{~nm}$ and $3.0 \mu \mathrm{m}$ at solar abundance, for IB model at ages 2, 5, 7.5, 10 and 20 Myr. Solid line shows cluster spectrum from our computations, whereas the dotted lines show the total cluster spectrum as computed by Mas-Hesse \& Kunth (1991). The rms of the differences between the two spectra over the wavelength range $136.2 \mathrm{~nm}$ to $2.2 \mu \mathrm{m}$ are $0.08,0.10,0.18,0.20$ and 0.08 dex for 2, 5, 7.5, 10 and 20 Myr respectively. Hence the agreement is generally good over the entire wavelength range at all comparison ages except at 7.5 and $10 \mathrm{Myr}$, at which time cluster is in its red supergiant phase. Considering that the spectral shape is rapidly changing during the red supergiant phase and that Mas-Hesse \& Kunth derived spectrum from available broad band photometry of red supergiants, the observed differences during this phase are not un-reasonable.

\section{Summary}

We have constructed an evolutionary population synthesis model incorporating the recently available stellar evolutionary models and model stellar atmospheres. We synthesize a number of observable quantities in the optical and near infrared part of the spectrum, suitable for comparison with the observed properties of giant star forming complexes. The ratio of 
$\mathrm{H} \beta$ to $B$ band luminosity, $B-V, V-R$ and $V-K$ colors have been studied as a function of cluster evolution for Instantaneous Burst and continuous star formation models. Computations performed for a twoburst model are also presented with the younger burst rich in massive stars capable of ionization, and the older burst rich in red supergiants. Color evolution shows an initial steady phase for both instantaneous burst and continuous star formation models, followed by a red bump between 5-15 Myr for instantaneous burst. In the observable plane, two-burst models occupy quite distinct regions compared to instantaneous burst and continuous star formation models. Dependence of computed quantities on the IMF parameters are also studied. Flat IMF is found to produce the bluest colors when the cluster is young, and the reddest colors when red supergiants start appearing in the cluster. The effect of the statistical fluctuations inherent to the process of star formation on the computed quantities are studied using Monte Carlo simulations and letting the cluster evolve upto $20 \mathrm{Myr}$. For GEHRs with $\mathrm{H} \alpha$ luminosities exceeding $10^{39} \mathrm{erg} \mathrm{s}^{-1}$, the statistical effects are found to be negligible. The spectra from our models are compared with the computations of Mas-Hesse \& Kunth (1991) at 2, 5, 7.5, 10 and $20 \mathrm{Myr}$, and the agreement is found to be reasonably good.

It is a pleasure to thank T.P. Prabhu, who took an active interest throughout the period of this work and contributed greatly through discussions, suggestions and later by critically reading several versions of the manuscript. A modelling of this kind is impossible without the availability of input data in electronic form. I thank G. Meynet and G. Schaller for providing access to their stellar evolutionary data through the network and M. Parthasarathy and S. Giridhar for provided me the data tapes containing new and old versions of stellar atmospheric models by Kurucz. I thank the two anonymous referees for their suggestions which helped in improving the manuscript enormously.

\section{REFERENCES}

Balzano, V. 1983, ApJ, 268, 602

Baschek, B., Scholz, M., \& Wehrse, R. 1991, A\&A, 246, 374

Beech, M., \& Mitalas, R. 1994, ApJS, 95, 517

Belfort, P., Mochkovitch, R., \& Dennefeld, M. 1987, A\&A, 176, 1

Bessell, M.S. 1979, PASP, 91, 589

Bessell, M.S. 1990, PASP, 102, 1181
Bessell, M.S., \& Brett, J.M. 1988, PASP, 100, 1134

Brown, R.L., \& Mathews, W.G. 1970, ApJ, 160, 939

Bruzual G.A., \& Charlot, S. 1993, ApJ, 405, 538

Buzzoni, A. 1989, ApJS, 71, 817

Dopita, M.A., \& Evans, I.N. 1986, ApJ, 307, 431

Dreiling, L.A., \& Bell, R.A. 1980, ApJ, 241, 736

Elias, J.H., Frogel, J.A., \& Humphreys, R.M. 1985, ApJS, 57, 91

Elmegreen, B.G. 1992, in Star Formation in Stellar Systems, editors Tenorio-Tagle, G., Prieto, M., \& Sánchez, F. (Cambridge Univ. Press) p. 409

Elmegreen, B.G., \& Lada, C.J. 1977, ApJ, 214, 725

Ezer, D., \& Cameron, A.G.W. 1967, Canadian J. Phys., 45, 3429

Ferland, G.J. 1980, PASP 92, 596

Flower, P.J. 1977, A\&A, 54, 31

Humphreys, R.M., \& McElroy, D.B. 1984 ApJ, 284, 565

Johnson, H.L, 1966, ARA\&A, 4, 193

Kudritzki, R.P., Pauldrach, A., Puls J., \& Abbott, D.C. 1989, A\&A, 219, 205

Kurucz, R.L. 1979, ApJS, 40, 1

Kurucz, R.L. 1992, in Stellar Populations of galaxies, edited by Barbuy, B. \& Renzini, A (Kluwer academic publishers, IAU 149), p. 225

Lang, K. R. 1992, Astrophysical Data: Planets and stars (Springer-Verlag, New York, Inc.) p.112

Larson, R.B., \& Tinsley, B.M. 1978, ApJ, 219, 46

Leitherer, C. 1990, ApJS, 73, 1

Leitherer, C., \& Heckman, T.M. 1995, ApJS, 96, 9

Lequeux, J., Maucherat-Joubert, M., Deharveng, J.M., \& Kunth, D. 1981, A\&A, 103, 305

Lequeux, J., Peimbert, M., Rayo, J.F., Serrano, A., \& Torres-Peimbert, S. 1979, A\&A, 80, 155

Maeder, A. 1990, A\&AS, 84, 139

Maeder, A., Meynet, G. 1989, A\&A, 210, 155

Mas-Hesse, M., \& Kunth, D. 1991 A\&AS, 88, 399

Mathis, J.S., Chu, Y.-H., \& Peterson, D.E. 1985, ApJ, 292, 155

Mayya, Y.D. 1994, AJ, 108, 1276

McCall, M.L., Rybski, P.M., \& Shields, G.A. 1985, ApJS, 57, 1

Melnick, J., Terlevich, R., \& Eggleton, P.P. 1985, MNRAS, 216, 255

Mezger, P.G. 1978, A\&A, 70, 565 
Olofsson, K. 1989, A\&AS, 80, 317

Osterbrock, D.E. 1974, Astrophysics of Gaseous Nebulae, Freeman, San Francisco

Osterbrock, D.E. 1989, Astrophysics of Gaseous Nebulae and Active Galactic Nuclei, Univ. Science Books, California

Panagia, N. 1973, AJ 78, 929

Rieke, G.H., Lebofsky, M.J., Thompson, R.I., Low, F.J., \& Tokunaga, A.T. 1980, ApJ, 238, 24

Rogers, F.J., \& Iglesias, C.A. 1992, ApJS, 79, 507

Rubin, R.H. 1985, ApJS, 57, 349

Scalo, J.M. 1987, in Starbursts and Galaxy Evolution, edited by T.X. Thuan, T. Montmerle \& J. T. T. Van (Editions Frontieres, Gif sur Yvette), p445

Schaller, G., Schaerer, G., Meynet, G., \& Maeder, A. 1992, A\&AS, 96, 269

Searle, L., Sargent, W.L.W., \& Bagnuolo, W.G. 1973, ApJ, 179, 427

Sérsic, J.L., \& Pastoriza, M. 1965, PASP, 77, 287.

Terlevich, R., Melnick, J., Masegosa, J., Moles, M., \& Copetti, M.V.F. 1991, A\&AS, 91, 285

This 2-column preprint was prepared with the AAS LATEX macros v3.0.

Fig. 1.- Lyman continuum photon rate at the stellar surface $\left(n_{\text {lyman }}\right)$, bolometric correction (BC), $B-V, V-R$, and $V-K$ colors of stars as computed by us using Schaller et al. (1992) and Kurucz (1992) models, are compared with observations in figures (a), (b), (c), (d) and (e) respectively as a function of $\mathrm{T}_{\text {eff }}$. Observational data are from Flower (1977) or Johnson (1966) as indicated, with filled circles and inverted triangles representing data for main-sequence and supergiants respectively. The computed quantities for main-sequence and supergiants are denoted by solid and dashed lines respectively. Lyman continuum surface fluxes are compared with the computations of Mas-Hesse \& Kunth (1991).
Fig. 2.- Equivalent width of $\mathrm{H} \beta$ emission line is plotted against the ratio of $\mathrm{H} \beta$ to blue band luminosity $\left(\frac{\phi}{L_{B}}\right)$ in $\log$-log plot. Age is the parameter which is varying along the locus, with successive dots separated by $0.5 \mathrm{Myr}$. The relationship is not very sensitive to IMF parameters or the scenario of star formation and hence is useful in converting one form of data to another.

Fig. 3.- Contribution to total luminosities from stars in four mass bins are plotted as a function of cluster age for an instantaneous burst with IMF parameters $m_{l}=1, m_{u}=60$, and slope $=2.5$. The chosen mass bins are: $40<m<60$ (dotted line), $15<m<40$ (dashed line), $5<m<15$ (dot-dashed), $m$ expressed in units of $M_{\odot}$. The contribution from the fourth bin $(1<m<5)$ lies below the plotted range. The quantities plotted are (a) bolometric luminosity, (b) lyman continuum photon rate, (c) $B$ (d) $V$ (e) $R$ and (f) $K$ band luminosities. The solid lines correspond to the summed contribution from all the stars which are again plotted in Fig. (g) for a direct comparison of band luminosities. The beginning and the end of the supergiant phases for selected masses are marked on this plot. Ordinates are in units of erg s s${ }^{-1} M_{\odot}{ }^{-1}$ for all plots except (b) where it is $\mathrm{ph} \mathrm{s}^{-1} M_{\odot}{ }^{-1}$. See section 3.4 for further details.

Fig. 4.- Evolution of (a) $\mathrm{H} \beta$ to $B$ band luminosity ratio $\left(\log \left(\frac{\phi}{L_{B}}\right)\right)$, (b) $B-V$, (c) $V-R$ and (d) $V-K$ colors for instantaneous burst and continuous star formation models are shown. Solid and dotted lines represent smoothed (over $0.5 \mathrm{Myr}$ ) and unsmoothed IB models. Dot-dashed and dashed lines represent CSF models with constant and exponentially decreasing (e-folding time scale of $10 \mathrm{Myr}$ ) star formation rates respectively. Note the initial steady phase, a red bump and another steady phase during the IB color evolution. The IMF used is the same as for Fig. 3 . 
Fig. 5.- $\log \left(\frac{\phi}{L_{B}}\right)$ is plotted against $B-V, V-R$ and $V-K$ colors in figs (a), (b) and (c) respectively. The dotted line is for the instantaneous burst with thick dots at every $0.5 \mathrm{Myr}$ interval. Solid lines identified by $0,3.5,5.0$ and 6.0 are tracks for models with coexisting stellar populations from two bursts of equal strengths. The labeled numbers denote the age of the younger of the two populations in Myr, whose positions are marked by the asterix on the IB model. The older population age varies along the track between 0 and 13 Myr. CSF model with constant star formation rate is also plotted (dashed line) for $20 \mathrm{Myr}$ of evolution. The IMF used is the same as for Fig. 3.

Fig. 6. - Evolution of (a) $\log \left(\frac{\phi}{L_{B}}\right)$, (b) $B-V$, (c) $V-R$ and (d) $V-K$ colors for IB models are shown for 3 IMFs. The parameters of IMF used are: $m_{l}=1, m_{u}=120$ and slope $=1$ (dotted line), $m_{l}=1$, $m_{u}=60$ and slope $=2.5$ (solid line) and $m_{l}=1$, $m_{u}=30$ and slope $=3.5$ (dashed line). Notice that as the massive star fraction increases in the cluster, colors become bluer in the early phase and redder after 5 Myr.

Fig. 7.- Same as Fig. 6, but for continuous star formation scenario with constant star formation rate.

Fig. 8. - $\log \left(\frac{\phi}{L_{B}}\right)$ is plotted against $B-V, V-R$ and $V-K$ colors in Figs (a), (b) and (c) respectively for the composite two-burst models for the three IMFs used in Figs 6 and 7. Burst parameters are identical to Fig. 5. Ages of the younger bursts in Myr are denoted close to the tracks. Thick dots are placed every $0.5 \mathrm{Myr}$ starting at $6 \mathrm{Myr}$. IMF differences stand out for composite models when the younger burst is younger than 3.5 Myr. Beyond this age differences tend to decrease especially for $V-K$ color.

Fig. 9.- Errors due to statistical fluctuations intrinsic to star formation process on bolometric, $\mathrm{H} \beta$, $B$ band and $K$ band luminosities are plotted for different cluster masses. Numbers on the curves represent mass of the clusters in units of $6641 M_{\odot}$, the mass containing one star earlier than $\mathrm{O} 4$ for the IMF with $m_{l}=1, m_{u}=120$ and slope $=2.5$. The errors are computed as the rms fluctuations on the quantities over 100 Monte Carlo simulations. See text for details.

Fig. 10.- Same as Fig. 9, but the errors on $\log \left(\frac{\phi}{L_{B}}\right.$ ), $B-V, V-R$ and $V-K$ colors are plotted.
Fig. 11.- Comparison of the cluster+nebular spectrum for an IMF with $m_{l}=2, m_{u}=120$ and slope $=2.0$ at ages 2, 5, 7.5, 10 and $20 \mathrm{Myr}$ are done in Figs (a), (b), (c), (d) and (e) respectively for the instantaneous burst model. Thick lines are from our computations, with dots corresponding to the wavelengths at which computations are performed. Dotted lines are from Mas-Hesse \& Kunth (1991). Agreement is good in general with differences exceeding 0.1 dex only at 7.5 and $10 \mathrm{Myr}$, at which stage cluster is dominated by fast evolving red supergiants. 